Prognosemarker, Therapie und Langzeitfolgen

\title{
Hochdruck in der Schwangerschaft: Droht eine Präeklampsie?
}

\author{
Pathophysiologisch steht bei der Präeklamspie eine plazentare Dysfunktion im Mittelpunkt - \\ mit erheblichen Gefahren für Mutter und Kind. Wie hoch das Präeklampsierisiko ist, lässt sich \\ am Verhältnis von zwei angiogenetischen Faktoren ablesen.
}

\begin{abstract}
- Bei 8-15\% aller Schwangerschaften tritt im Verlauf eine Hypertonie erstmals auf oder eine vorbestehende verschlechtert sich. Dies bringt maternale und fetale Risiken mit sich wie Organversagen, disseminierte intravaskuläre Koagulation, Wachstumsretardierung, Frühgeburtlichkeit und intrauterinen Tod. Ein Viertel aller pränatalen Krankenhauseinweisungen sind einer Hypertonie zuzuschreiben, wie Prof. Vera RegitzZagrosek, Charité Berlin, ausführte.
\end{abstract}

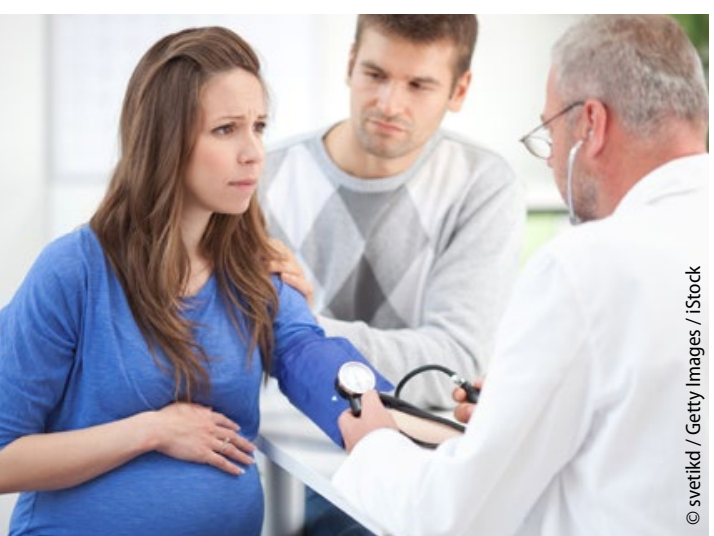

Grund zur Sorge: Blutdruck plötzlich zu hoch.

\section{Neuer prognostischer Marker}

Die Präeklampsie, eine De-novo-Hypertonie in der Schwangerschaft mit neuer signifikanter Proteinurie, kompliziert 5-7\% aller Schwangerschaften und manifestiert sich meist ab der Mitte der Gravidität. Pathogenetisch spielen angiogenetische Faktoren eine große Rolle, zum einen die Tyrosinkinase sFlt, die VEGF-Rezeptoren imitiert und den für die Neoangiogenese wichtigen VEGF (vascular endothelial growth factor) ab- fängt, und zum anderen den plazentaren Wachstumsfaktor (PlGF). Das Verhältnis dieser beiden Faktoren ist bei Frauen, die eine Präeklampsie entwickeln, gestört: Man beobachtet eine Verschiebung zu hoher sFlt- und niedriger PlGFKonzentration.

Neue Untersuchungen zeigen, dass sich das Präeklampsierisiko durch zusätzliche Bestimmung des sFlt/PlGFQuotienten noch sicherer vorhersagen lässt, als dies mit den Parametern Blutdruck und Proteinurie allein möglich ist. Regitz-Zagrosek erwartet, dass dieser Marker in der künftigen Leitlinienanpassung berücksichtigt werden wird.

\section{Präeklampsie vorbeugen}

Das Präeklampsierisiko kann jedoch durch Supplementation von $1 \mathrm{~g}$ Calcium pro Tag und niedrig dosiertes ASS vermindert werden. Eine pharmakologische Therapie der Schwangerschafts-Hypertonie ist erst bei systolischen Blutdruckwerten $>150 \mathrm{mmHg}$ oder diastolischen Werten $>99 \mathrm{mHg}$ in Erwägung zu ziehen. Eine Schwangere mit schwerer Hypertonie $(\geq 170 / 110 \mathrm{mmHg})$ muss notfallmäBig ins Krankenhaus eingewiesen werden.

Von den Antihypertensiva kommen Alpha-Methyldopa, Metoprolol oder Labetalol, Nifedipin, Magnesiumsulfat oder Urapidil in der Schwangerschaft in Betracht. Strikt kontraindiziert sind dagegen ACE-Hemmer, $\mathrm{AT}_{1}$-Rezeptorblocker und direkte Renin-Inhibitoren. Diuretika sollten möglichst auch vermieden werden, weil sie den plazentaren Blutfluss einschränken. Wie eine aktuelle Studie zeigt, bringt eine sehr strenge Blutdruckeinstellung in der Schwangerschaft keinen signifikanten Vorteil in der
Häufigkeit maternaler und fetaler Komplikationen im Vergleich zu einer weniger strengen.

\section{Niereninsuffizienz schreitet voran}

Langfristig ist zu beachten, dass Frauen mit Präeklampsie ein deutlich erhöhtes Risiko für spätere kardiovaskuläre Erkrankungen haben, wie Regitz-Zagrosek betonte. Die Präeklampsie stellt auch einen Risikofaktor für die Entwicklung einer Niereninsuffizienz dar, erklärte PD Dr. Christian S. Haas, Medizinische Universitätsklinik Lübeck. Der sFlt/ PlGF-Quotient kann hilfreich sein, um eine Präeklampsie von einer Niereninsuffizienz abzugrenzen.

Unabhängig von Hypertonie oder Präeklampsie besteht in der Schwangerschaft ein erhöhtes Risiko für die Progredienz einer vorbestehenden Niereninsuffizienz. Und diese wiederum erhöht das Risiko für fetale Komplikationen. Das Blutdruckziel < 140/90 mmHg sollte deshalb bei Frauen mit Niereninsuffizienz in der Schwangerschaft etwas strenger verfolgt werden als bei Frauen ohne Niereninsuffizienz.

Diuretika können bei Niereninsuffizienz eingesetzt werden, aber eine Hypovolämie muss vermieden werden. Auch eine Hypokalzämie sollte nicht auftreten. Da die Patientinnen häufig eine renale Anämie aufweisen, muss der Hämoglobinwert gut eingestellt werden auf $>10 \mathrm{~g} / \mathrm{dl}$. Wichtig ist eine ausreichende Substitution mit Eisen und Folsäure. Der Bedarf an Erythropoetin kann in der Schwangerschaft erhöht sein.

Dr. med. Angelika Bischoff

- Symposium "Schwangerschaft und internistische Medikation", DGIM-Kongress, 11. April 2016 